\title{
ALEA - Adaptive eLEArning System - Lernende datenbankbasierte Lernsysteme in der Datenbanklehre
}

\section{Datenbank Spektrum}

\author{
Kerstin Schneider ${ }^{1} \mathbb{D} \cdot$ Fabian Keller $^{1}$ Pia Habekost ${ }^{1} \cdot$ Victor Schmeil $^{1}$ Markus Dampmann ${ }^{1}$ Florian Schorch ${ }^{1}$
}

Eingegangen: 10. Oktober 2020 / Angenommen: 15. Mai 2021 / Online publiziert: 22. Juni 2021

(c) Der/die Autor(en) 2021

\section{Zusammenfassung}

Im Bereich Datenbanken werden selbstentwickelte E-Learning-Systeme an vielen Hochschulen seit Jahren erfolgreich verwendet. An der Hochschule Harz werden E-Learning-Systeme im Bereich Datenbanken im Rahmen der Lehre und für die Lehre entwickelt, weiterentwickelt und eingesetzt. Das Gesamtsystem, welches die zusammengehörenden Systeme umfasst, wird als ALEA bezeichnet. Es werden relevante Komponenten von ALEA erläutert, die im Rahmen der Datenbanklehre für die klassischen Teilgebiete SQL, ER-to-Relational-Mapping und Normalisierung genutzt werden.

Schlüsselwörter E-Learning $\cdot$ Recommender Systems $\cdot$ SQL $\cdot$ ER-to-Relational-Mapping $\cdot$ Normalformen

\section{Einleitung}

Der Einsatz von E-Learning-Systemen ist zunehmend verbreitet. Gerade der Bereich Datenbanken hat den interessanten Aspekt, dass die verwendeten E-Learning-Systeme selbst wiederum Teil der Lehrinhalte sind, da diese i. d. R. klassische datenbankbasierte Systeme darstellen. Aspekte wie Analysen des Lernverhaltens mit dem Ziel der Optimierung der Lernprozesse betreffen ein Kernthema von Datenbanken wie bspw. auch die Erzeugung von Empfehlungen von Aufgaben oder Hilfetexten mit einem speicherbasierten Ansatz.

Daher ist es auch nicht überraschend, dass gerade im Bereich Datenbanken selbstentwickelte E-Learning-Systeme an vielen Hochschulen seit vielen Jahren erfolgreich genutzt werden. An der Hochschule Harz werden E-Learning-Systeme im Bereich Datenbanken seit 13 Jahren im Rahmen der Lehre und für die Lehre entwickelt, weiterentwickelt und eingesetzt. Im Folgenden werden nach einer

Kerstin Schneider

kschneider@hs-harz.de

1 Hochschule Harz Wernigerode, Friedrichstraße 57-59, 38855 Wernigerode, Deutschland
Übersicht über E-Learning-Systeme im Bereich Datenbanken relevante Komponenten von ALEA erläutert.

\section{E-Learning in der Lehre}

Das Lernen der theoretischen und anwendungsbezogenen Datenbank-Themen ist effektiver, effizienter und durchdringender möglich, wenn diese Lehrinhalte praktisch erfahren und geübt werden können, so dass sich die Ergänzung der Lehre im Bereich Datenbanken durch eigenentwickelte datenbankbasierte E-Learning-Systeme nicht nur an der Hochschule Harz als sehr nützlich erwiesen hat. Viele Aspekte bei der Entwicklung dieser Systeme fallen in den Bereich Datenbanken, so dass zudem die Erkenntnisse über die Systeme selbst die Datenbank-Lehre sinnvoll ergänzen.

Das eLearning Datenbank Portal edb-Portal der TH Köln [7-9] ist ein sehr bekanntes Beispiel für ein umfangreiches E-Learning-Angebot für den Bereich Datenbanken, welches sich aus vielfältigen Beiträgen verschiedener Hochschulen zusammensetzt. Hier wurde schon sehr früh begonnen, E-Learning-Anwendungen aufzubauen (siehe [18]). Es enthält zurzeit verschiedene Anwendungen: MCT (Multiple-Choice-Test), SQL-Trainer, 3NF-Trainer, ER-Trainer, SQL-Puzzle, PL/SQL-Trainer sowie das RelaX-System [13, 24]. Es wird ergänzt durch ein Wiki. 
Ein anderes Beispiel sind die Anwendungen an der TU München: DB-Normalizer [2], SQL-Schnittstelle zu Hyper [12] und das IRA-Tool für relationale Algebra [17]. Diese E-Learning-Anwendungen adressieren die grundlegenden Themen, welche die Datenbanklehre beinhalten sollte: ERM, Normalisierung, relationale Algebra sowie SQL und PL/SQL.

Die Anfragesprache SQL wurde entworfen mit der Zielsetzung, dass die Sprache von den Anwendenden leicht zu erlernen ist, um bspw. Fehler zu reduzieren. Nichtsdestotrotz erfordert es einige Übung, diese Anfragesprache zu erlernen und eine Sprache lernt sich sehr gut durch Sprechen. Daher entstanden hier auch weitere Lernsysteme. Bekannte Beispiele sind SQL Island [22] für das spielerische Erlernen der Sprache SQL und ein SQL-Hörbuch [19] mit einem ganz neuen Ansatz über das Hören. Kompetenzen im Bereich Datenmodellierung bleiben weiterhin sehr wichtig, auch wenn in manchen Datenbanken Daten schemalos verwaltet werden. Mit dem MonstER Park [20, 21] kann ERModellierung und Mapping spielerisch erlernt werden.

$\mathrm{Zu}$ nennen ist auch ein Ansatz des blended-Learning, welches das Selbstlernen der Grundlagen über bereitgestellte Videos mit ergänzendem Buch vor dem Besuch der Lehrveranstaltung erfordert $[5,6]$. Ebenso sind hier die blendedLearning-Vorlesungen mit Videos und den grafischen Fachlandkarten zu nennen [16, 25]. Zudem sind mit E-LearningUmgebungen wie ILIAS [11] eigene Lernmodule durch die Lehrenden erstellbar. ILIAS ermöglicht auch Funktionen für Learning Analytics.

Das ALEA-System adressiert die gleichen Themengebiete, jedoch mit unterschiedlichen Anwendungen. Die Benutzungsoberflächen wurden und werden weiterentwickelt nach den Erfahrungen und Bedürfnissen der Lernenden und die vielfältigen Interaktionsmöglichkeiten wurden nach und nach integriert und ergänzt. Es entstanden und entstehen kurze Videos (bspw. Screencasts) sowie Aufgabenstellungen, Hilfetexte unter starker Mitwirkung der Studierenden [23]. Allein die Korrektur oder Verschönerung einer Aufgabenstellung im System durch die Tutoren oder Lehrpersonen (dargestellt am Beamer), welche sich sofort bei allen Studierenden auswirkt, kann das Grundverständnis für datenbankbasierte Systeme deutlich erhöhen.

In ALEA sind die Komponenten so angelegt, dass die Themen, Fragen, Labore und Challenges mit den vorbereiteten Hinweisen und Hilfestellungen zunehmend leichter administrierbar und ergänzbar sind. Auf der anwachsenden integrierten Datenbasis soll Learning Analytics verstärkt angewendet werden. Idealerweise wird ALEA intelligenter. Hilfestellungen wie Videos, Podcasts, Grafiken und Texte sollen vermehrt neben den vordefinierten Zuordnungen empfohlen werden.

\section{Das ALEA-System}

Erste Versionen des E-Learning-Systems wurden bereits 2008 [10, 15] als Webanwendung (Apache Tomcat, J2EE, MVC-Architektur) unter dem Namen ELEA insbesondere für das Lernen von SQL entwickelt. In den folgenden Jahren wurden weitere Anwendungen und mobile Apps im Rahmen von studentischen Arbeiten erstellt. Eine Projektarbeit 2015-2016 [14] legte den Fokus bei einer Neuentwicklung des Systems neben der erweiterten Benutzungsschnittstelle zur Bearbeitung der Aufgaben besonders auf die Verwaltung und Administration der Labore und Aufgaben unter Einbindung von unterschiedlichen Datenbankschemas. Die Verwaltung musste somit nicht mehr direkt über das Datenbanksystem durchgeführt werden. Die Darstellung der Ergebnisse der Studierenden in den Laboren war nun live präsentierbar.

Für eine bessere Unterstützung der Selbstlernphasen wurde der Bedarf an vielfältigen Möglichkeiten von Fragen und Feedback der Lernenden im Kontext der Aufgaben sichtbar. Insbesondere in den berufsbegleitenden Studiengängen ist die entfernte oder asynchrone Kommunikation mit den Lehrpersonen häufig notwendig. Bei der Integration zusätzlicher Interaktionsarten im Jahr 2018 wurde ersichtlich, dass Anpassungen nicht einfach möglich sind. Das System sollte jedoch aufgrund seiner Vorteile im Einsatz bleiben.

Erweiterungen des Systems wurde daher seitdem auf einer überwiegend gemeinsamen Datenbasis durch Ergänzung von Webanwendungen durchgeführt, die auf Basis der in Vertiefungsvorlesungen im Bereich Datenbanken genutzten Technologie entwickelt werden (Apache Tomcat, MVC-Architektur, J2EE, CSS, JS, ajax, JQuery, usw.) und möglichst wenige Frameworks nutzen. Diese Anwendungen legen den Fokus auf die Erhöhung der Lernunterstützung, Interaktionen und auf Erweiterungen der Hilfestellungen. Vielfältige multimediale Elemente sollen für die Studierenden vorbereitet, zugeordnet oder durch das System empfohlen werden. Neben den studentischen Arbeiten, welche im Rahmen der Datenbanklehre oder als Bacheloroder Masterarbeiten durchgeführt werden, wurde dies auch als Teilprojekt im DigEL-Projekt „Digitalisierte Elemente in der Lehre“ zur Erarbeitung einer E-Learning-Strategie für die Hochschule Harz 2017-2019 durchgeführt. Neuere Komponenten des Systems ermöglichen eine gemeinsame Nutzung von Teilkomponenten über Challenges, die aus unterschiedlichen Fragetypen mit verschiedenen Benutzungsoberflächen bestehen. Die Administration und Verwaltung einzelner neuartiger Aufgabenarten und multimedialer Informationen wird teilweise noch direkt von Lehrpersonen über die Datenbank durchgeführt, was wiederum als Teil der Datenbanklehre angesehen werden kann. Das betrifft bspw. das Einfügen von Bildern, Videos oder vordefinierte 
Zuordnungen von Hilfetexten zu Aufgaben. Durch intelligente Komponenten können Zuordnungen auf der Datenbasis ergänzt werden. Die gemeinsame Datenbasis soll Learning Analytics ermöglichen. Das Gesamtsystem wird ALEA genannt.

\section{ALEA SQL}

ALEA SQL ist eine Webanwendung, die das Erlernen von SQL-Anfragen (Queries) unterstützt. Studierenden werden Labore zur Bearbeitung zugewiesen, die eine zuvor zugeordnete Menge von Aufgaben enthalten. Erfolgreich bearbeitete Aufgaben werden aus den Laboren entfernt.

$\mathrm{Zu}$ einer $\mathrm{zu}$ lösenden Aufgabe wird neben einer textuellen Beschreibung eine Voransicht der erwünschten Ergebnistabelle, die durch eine zu der jeweiligen Aufgabe in der Datenbank enthaltenen Query erzeugt wird, angezeigt (siehe Abb. 1). Über die Buttons auf der rechten Seite (siehe Abb. 1) lassen sich Informationen zum aktuellen Schema anzeigen: eine Übersicht aller Tabellen mit Spaltennamen und Datentypen, eine Liste aller Fremdschlüsselbeziehungen, Abbildungen des konzeptuellen, logischen und physischen Datenmodells sowie dem Labor zugeordnete Screen- casts. Die Buttons auf der linken Seite sind für den Aufgaben zugeordnete oder empfohlene Hilfetexte, Podcasts, Videos und Grafiken vorgesehen.

Nach dem Absenden der Anfrage zur Lösung der Aufgabe werden zum Vergleich beide Tabellen angezeigt. Falls die eingegebene SQL-Anfrage nicht die gewünschte Lösung erzeugt, können die Lernenden diese, da sie zur weiteren Bearbeitung erhalten bleibt, anpassen und sich somit nach und nach der Lösung annähern. Im Bereich ,Zusatzinformationen“ werden Hinweise zu Fehlerursachen angezeigt (siehe Abb. 2). Es besteht die Möglichkeit, eine Frage im Kontext der aktuellen Bearbeitung der Aufgabe an die Tutoren zu hinterlassen (siehe Abb. 2). Eine Aufgabe kann für eine spätere Bearbeitung zurückgestellt und übersprungen werden.

Sollten die Ergebnistabellen übereinstimmen, wird mit dem Hinweis „Die Anfrage liefert das korrekte Ergebnis“ die Aufgabe als gelöst aus dem Labor entfernt (siehe Abb. 3). Da die Anfrage trotzdem für die Aufgabe nicht richtig sein könnte, können die Tutoren die Lösungen auf der Datenbank prüfen und den Lernenden falls notwendig weitere Hinweise geben. Die Lernenden können direkte Erkenntnisse über den Bezug zu Relationen als Mengen von Tupeln gewinnen. Wenn die Aufgabe keine Sortierung
Abb. 1 SQL - Beispielaufgabe (Ausschnitt)

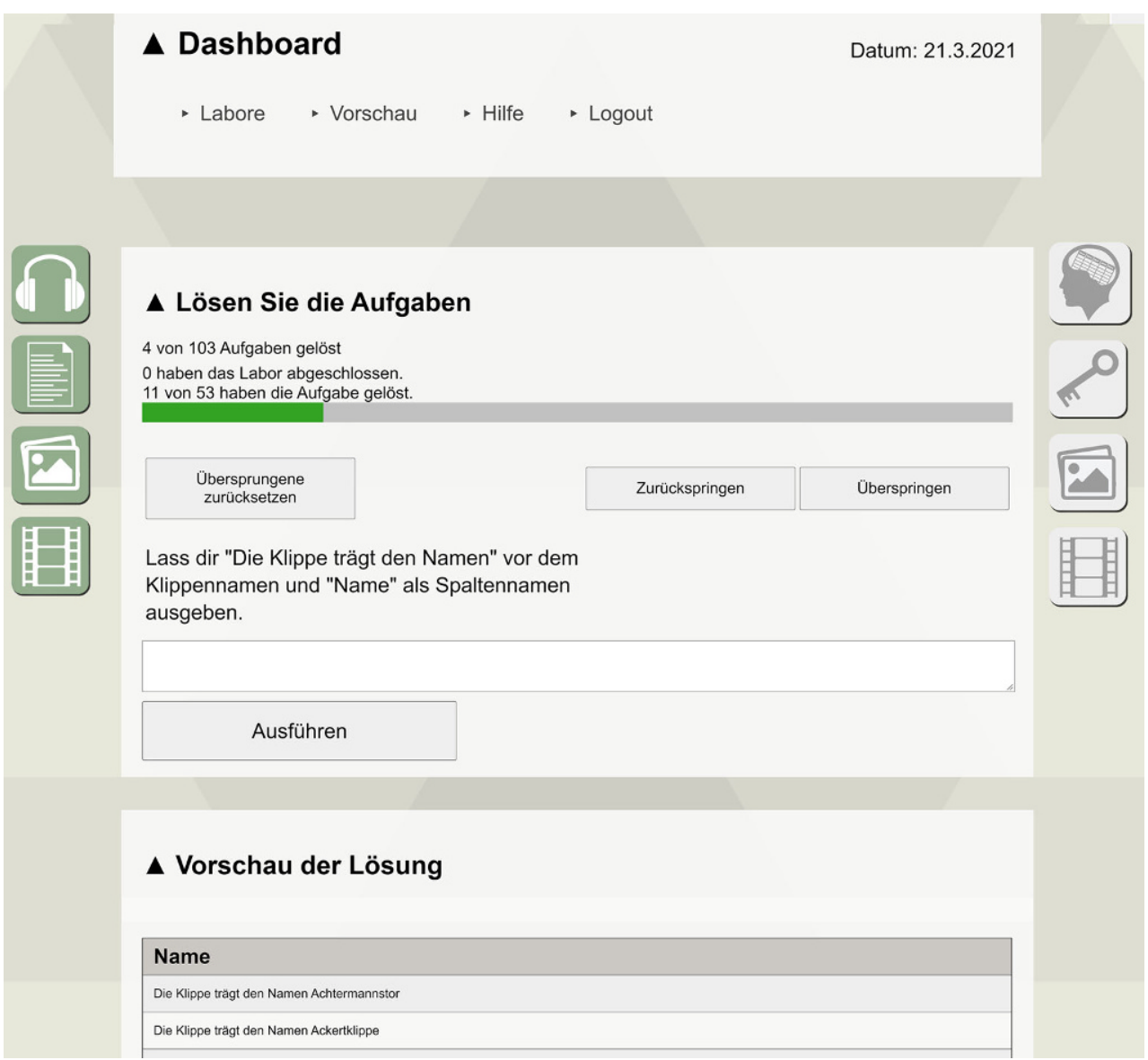


Abb. 2 SQL - Nicht korrekte Ausgabe mit Hinweisen auf den Fehler (Ausschnitt)

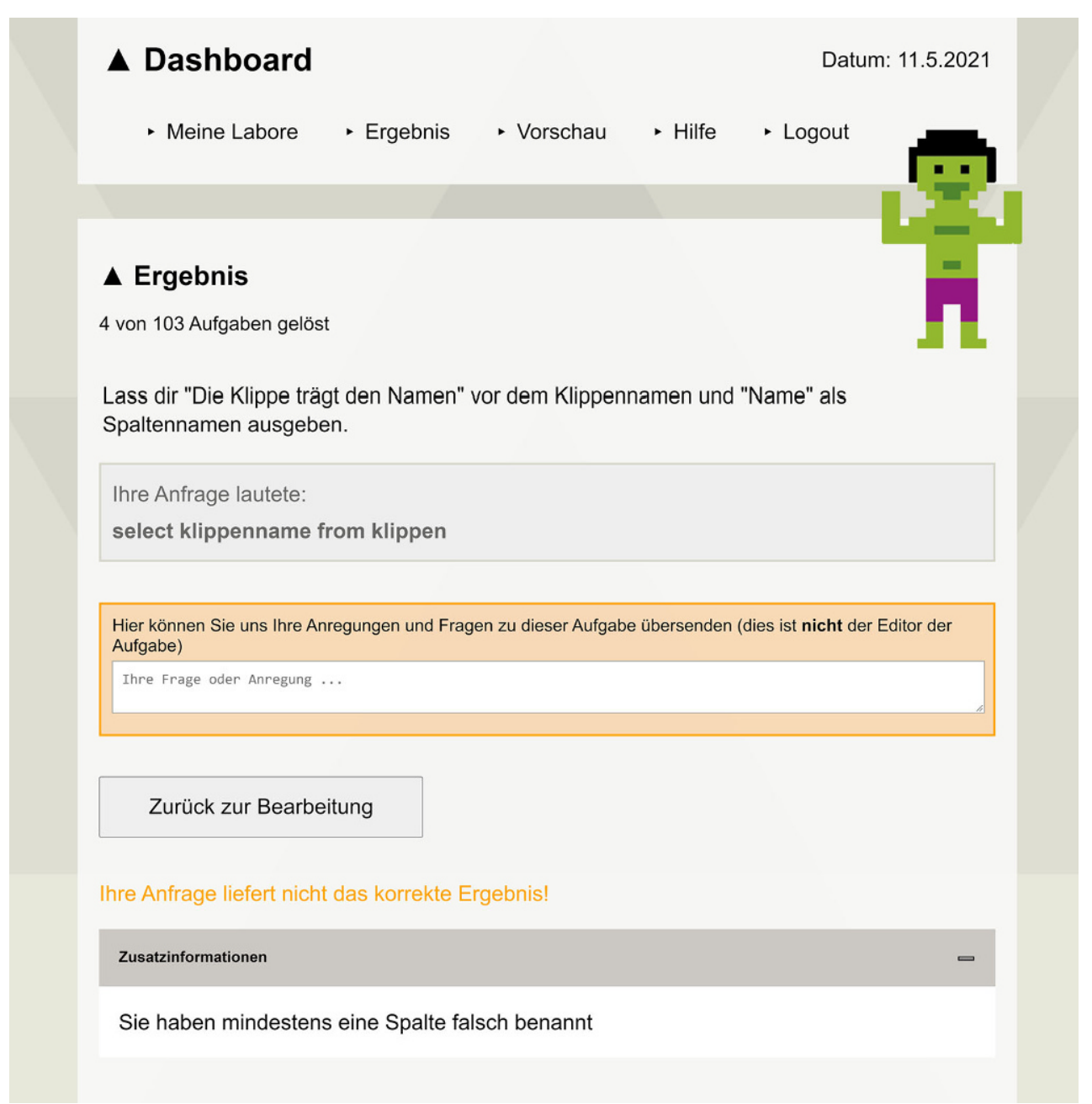

der Ergebnismenge erfordert, wird das Ergebnis als korrekt angesehen, auch wenn die Ergebnistabellen unterschiedlich sortiert sind. Ebenso können Ergebnistabellen mit unterschiedlicher Anzahl Zeilen übereinstimmen, wenn doppelte Zeilen nicht explizit entfernt werden sollen. Die Lernenden haben, bevor sie zur nächsten Aufgabe übergehen, die Möglichkeit, Anregungen zu übermitteln. Zudem erscheint hier ein 5-Sterne-Bewertungssystem, mit dem die Lernenden die Aufgabe bewerten müssen. Welche Erkenntnisse sich aus den Bewertungen ergeben, steht noch nicht fest.

Ein kompetitiver Nebeneffekt entsteht durch die Anzeige der Anzahl der Lernenden, welche das aktuelle Labor bereits beendet haben. Zudem wird angezeigt, wie viele Lernende die aktuelle Aufgabe bereits erfolgreich abgeschlossen haben.

ALEA SQL wird auch für komplexe Anfragen genutzt, so dass es ebenso in Vertiefungsvorlesungen eingesetzt wird. Analytische Anfragen u. a. auf Star- oder SnowflakeSchemas sind möglich. Hier wird ggfs. die Darstellung der Ergebnismenge mit einer Scroll-Leiste versehen oder falls notwendig auf einen sinnvollen Ausschnitt beschränkt und nur teilweise dargestellt. Die Queries der Lernenden können sehr umfangreiche Ergebnisse erzeugen, die nicht vollständig dargestellt werden müssen. Zudem können Aufgabenarten und Labore auf unterschiedlich starke Datenbankserver geleitet werden. Es werden verschiedene Datenbanksysteme eingesetzt. Es gelten unterschiedliche Lizenzmodelle und Zugangskonzepte. ALEA ist daher teilweise lediglich im Intranet zugänglich.

\section{ALEA Mapping}

ER-to-Relational-Mapping gehört zu klassischen Aufgaben, die in einführenden Datenbankvorlesungen gelehrt werden. Die Komponente ALEA Mapping addressiert diesen Aufgabenbereich. Die Studierenden sollen die Möglichkeit erhalten, dieses Mapping intensiv zu üben. Dadurch soll das Verständnis für Konzeptuelle Datenmodelle, wie ER-Modelle, vertieft und der Aufbau von Tabellen mit den Integritätsbedingungen (Not Null, Unique, Primary Key, Foreign Key) verinnerlicht werden. Gleichzeitig werden 
Abb. 3 SQL - Gelöste Aufgabe

D Dashboard
- Meine Labore - Ergebnis , Vorschau , Hilfe , Logout

\section{$\Delta$ Ergebnis}

4 von 103 Aufgaben gelöst

Lass dir "Die Klippe trägt den Namen" vor dem Klippennamen und "Name" als Spaltennamen ausgeben.

Ihre Anfrage lautete:

select 'Die Klippe trägt den Namen ' || klippenname "Name" from klippen

\section{Ihre Bewertung}

$\star \star \star \star \star \star ⿱ 乛 ⿻ ⺀ 大$

Weiter

Ihre Anfrage liefert das korrekte Ergebnis!

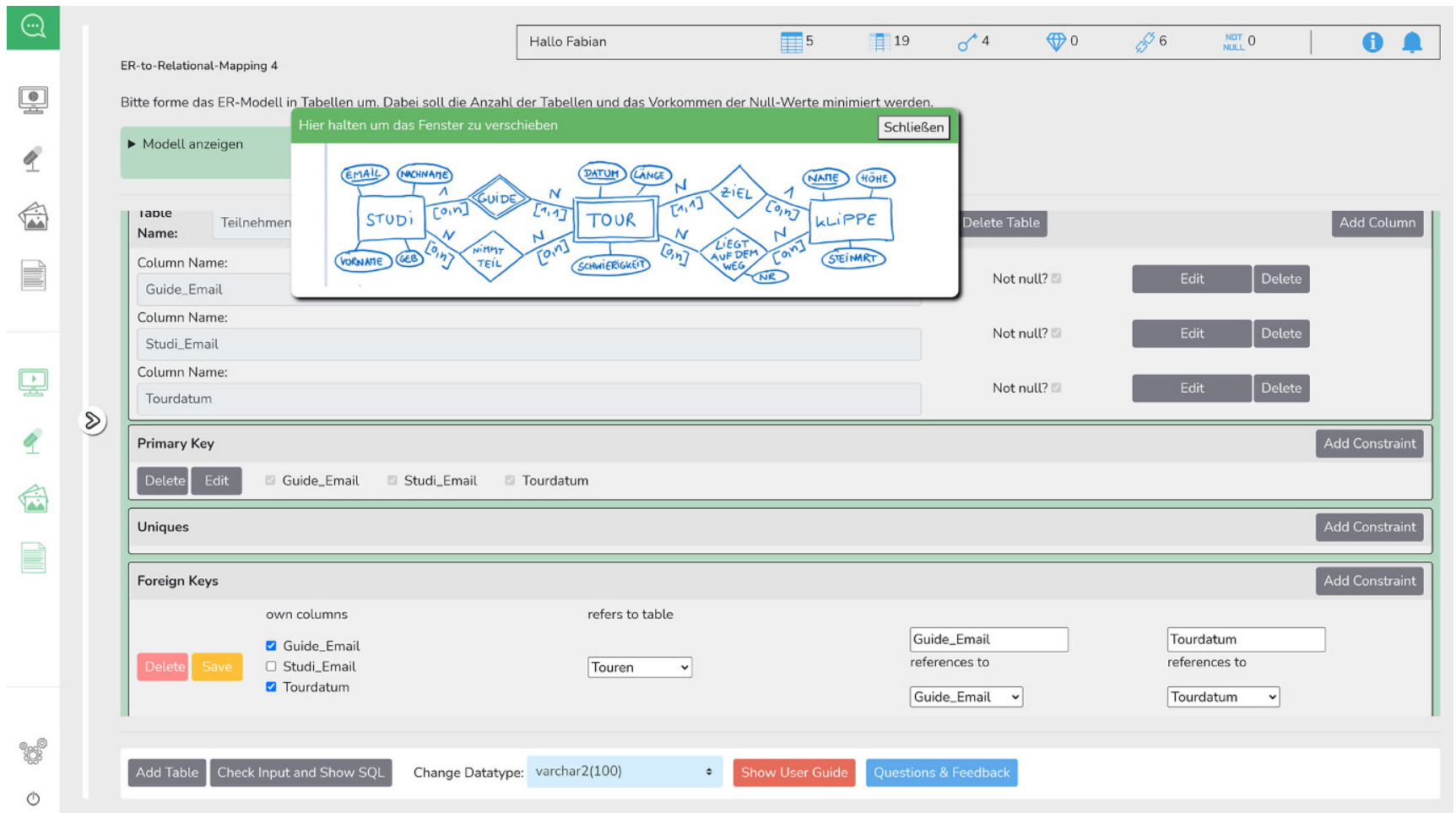

Abb. 4 Mapping (Ausschnitt)

Regeln und Strukturen von DDL-Anweisungen erlernt. Von Aspekten wie Check-Constraints und unterschiedlichen Datentypen wird abstrahiert, da diese im Rahmen dieser Aufgaben nicht relevant sind.
Es ist wichtig, dass hierbei notwendige Korrektur- und Feedbackleistungen der Tutoren minimiert werden, um diesen die Zeit für andere relevante Themen zu erhalten. $\mathrm{Zu}$ dem soll die Komponente auch in Selbstlernphasen sinnvoll eingesetzt werden können. Die Eingaben der Studieren- 
den werden weitestgehend vom System auf Fehler geprüft. Wenn möglich soll das System Hilfestellungen geben. Die resultierenden Lösungsvorschläge der Studierenden enthalten keine automatisch erkennbaren Fehler und liegen in einem ausführbaren DDL-Format vor.

Die Aufgaben in dieser Komponente enthalten Abbildungen von Konzeptuellen Modellen in unterschiedlichen Notationen, beispielsweise gezeichnete ER-Modelle in der Chen-Notation [4] (siehe Abb. 4). Die in der Aufgabe dargestellten ER-Modelle sind auf ein Relationenmodell bzw. relationales Modell abzubilden, indem die entsprechenden Relationen, Attribute und Bedingungen in Form von Tabellen, Spalten und Integritätsbedingungen definiert werden.

In den Aufgaben können unterschiedliche Anforderungen bezogen auf die Minimierung der Anzahl von Tabellen und/oder Null-Werten angegeben sein, so dass $\mathrm{zu}$ einer Abbildung unterschiedliche Lösungen gehören können, auch wenn bereits die Benennung der Tabellen und Spalten nicht berücksichtigt wird. Werden diese Bezeichnungen nicht berücksichtigt, können für manche Abbildungen für dieselbe Aufgabenstellung mit identischen Anforderungen noch mehrere Lösungen existieren. Es ist jedoch möglich, didaktisch sinnvolle Aufgaben zu erstellen, welche die automatische Prüfung für bestimmte Aspekte erleichtern und somit für Selbstlernphasen förderlich sind.

Das System enthält Informationen zu der Lösung, wie bspw. die Anzahl der Tabellen, der Spalten oder der Fremdschlüssel, so dass die Lösung neben den Aspekten zu den inhärenten Regeln des Relationenmodells, bspw. ein Fremd- schlüssel kann lediglich auf einen Kandidatenschlüssel verweisen, bereits teilweise durch das System geprüft werden kann. Die allgemeinen Regeln, die sich auf die Struktur des Tabellenmodells oder der DDL-Anweisungen beziehen, sollen zudem weitestgehend bereits während der Eingabe der Lösung erlernt werden. Daher ist die Benutzungsoberfläche so gestaltet, dass die Eingaben bereits die Einhaltung ausgwählter Regeln erzwingen.

Über die visuelle Benutzungsoberfläche können die Lernenden Tabellen, Spalten und Integritätsbedingungen durch einen Klick hinzufügen und so definieren, wie es die Aufgabenstellung erfordert. Hierbei dienen „Save“ und „Edit“ Buttons als Unterstützung. Wird ein Element erstellt oder editiert, werden alle anderen Buttons deaktiviert. Gewisse Abhängigkeiten werden so automatisch erzwungen und mit Systemmeldungen ergänzt. Treten bei der Erstellung von Elementen Fehler auf, bspw. ein Spaltenname soll erneut in der Tabelle verwendet werden oder Spaltennamen enthalten unerlaubte Zeichen, wird die Eingabe geprüft, ohne dass sie zum Server gesendet wird.

Ein weiteres Ziel von ALEA Mapping ist es, die grundlegende Struktur von SQL-DDL-Anweisungen, zu verdeutlichen, die erforderlich sind, um die notwendigen Tabellen zu erstellen. Die Lernenden können sich den SQL-Code anzeigen lassen (Abb. 5). Die notwendige Zeichensetzung wie ";" oder “( )" wird hierbei vom System ergänzt, so dass die Lernenden sich auf die Zusammenhänge und Abhängigkeiten konzentrieren können, die in dieser Aufgabe im Vordergrund stehen.
Abb. 5 Mapping SQL-Code (Ausschnitt)
Test on Database Copy Code Save to Script

CREATE TABLE Studis (

Email varchar2(100) NOT NULL,

Vorname varchar2(100),

Nachname varchar2(100),

Geburtsdatum varchar2(100),

CONSTRAINT PK_Studis PRIMARY KEY ( Email )

);

CREATE TABLE Klippen (

Klippenname varchar2(100) NOT NULL,

Steinart varchar2(100),

Hoehe varchar2(100),

CONSTRAINT PK_Klippen PRIMARY KEY ( Klippenname )

);

CREATE TABLE Touren (

Guide_Email varchar2(100) NOT NULL,

Tourdatum varchar2(100) NOT NULL ,

Laenge varchar2(100),

Schwierigkeit varchar2(100),

Zielklippe varchar2(100),

CONSTRAINT PK_Touren PRIMARY KEY ( Guide_Email, Tourdatum ),

CONSTRAINT FK Touren 1 FOREIGN KEY (Guide Email ) REFERENCES Studis ( Email )

Feedback: Der SQL Code kann ausgeführt werden | Zu viele Tabellen! 
Der beim Lösen der Aufgabe entstandene DDL-Code kann innerhalb des Systems an das Datenbanksystem übermittelt und geprüft werden. Zudem werden Prüfungen über die zur Lösung vorliegendenden Informationen durch ALEA durchgeführt. Die Fehlermeldungen des Datenbanksystems als auch Feedback zu den Ergebnissen der zusätzlichen Prüfungen werden angezeigt. Darüber hinaus können sich die Lernenden den entstandenen Code für eine spätere Verwendung als DDL-Skript abspeichern. Da Datentypen im Rahmen dieser Aufgabe nicht relevant sind, werden die Datentypen aller Spalten auf variable Zeichenketten gesetzt. Die unterschiedliche Ausprägungen bei DBMS, bspw. varchar2, varchar oder vchar, lassen sich auswählen (siehe Abb. 4).

Im ALEA Mapping kann eine Lösung nicht eindeutig definiert und verglichen werden. Für eine Kontrolle durch das System zur Generierung von Feedback für die Lernenden in Abhängigkeit vom Nutzungskontext (bspw. Testat oder Übung) oder für die spätere Korrektur der Aufgaben durch die Lehrenden können Daten zur jeweiligen Aufgabe hinterlegt sein. Dies bezieht sich u.a. auf Information zur Anzahl der Tabellen, Primärschlüssel, Fremdschlüssel, Not-Null-Constraints außerhalb von Primärschlüsseln und Unique-Constraints sowie die Anzahl der jeweiligen Attribute auf diese Teile bezogen sowie insgesamt. Sollte die Aufgabe auf diese Aspekte bezogen mehrere Lösungen haben, kann zumindest jeweils mit den korrekten Bereichen gearbeitet werden.

Ein Beispiel ist die Anzahl der Unique-Contraints, die für eine richtige Lösung notwendig sind. Bspw. gibt die Lehrperson beim Erstellen der Aufgabe an, dass die Lösung genau ein Unique-Constraint beinhalten muss, da eine One-to-One-Relationship enthalten ist, aber ansonsten keine für ein Unique-Constraint bedeutsame Information. Allerdings ist die Anzahl der Attribute innerhalb des UniqueConstraints in der Lösung nicht exakt definierbar, wenn die Relationship-Menge in beiden Richtungen keine verpflichtende Kardinalitätsrestriktion hat und die Primärschlüssel der beiden beteiligten Entitätsmengen eine unterschiedliche Anzahl an Attributen enthalten.

In Abb. 4 ist für keine Spalte außerhalb der Primärschlüssel die Integritätsbedingung NOT NULL definiert worden (siehe oben rechts: NOT NULL 0). Der Fremdschlüssel in der Tabelle Touren, welcher auf die Zielklippe, d.h. auf den Klippennamen in der Tabelle Klippen, verweist, darf aber nicht NULL sein, daher ist die eingegebene Lösung offensichtlich noch nicht korrekt.

Senden die Lernenden ihre Lösungen ab, können durch das System bereits Überprüfungen erfolgen. Eine manuelle Kontrolle bleibt letztendlich wichtig. Da die Lösungen als Daten in Datenbanken gespeichert werden, sind hier wiederum vielfältige Möglichkeiten zur Entwicklung von datenbankbasierten Systemkomponenten zur Unterstützung gegeben. Auf die spezifischen Anforderungen der jeweiligen Aufgabe bezogenes Feedback des Systems wird in Abhängigkeit vom Kontext bspw. Selbstlernphase, Übung, Testat, Prüfungssituation angepasst.

Im Rahmen von Übungen oder Testaten ist es sinnvoll den Studierenden Aufgaben zuzuordnen, welche mit möglichst weitestgehendem Feedback des Systems gelöst werden müssen, so dass die abgegebenen Lösungen keine automatisch erkennbaren Fehler mehr enthalten sollten. $\mathrm{Zu}$ dem können Fragen der Lernenden von den Lehrpersonen im Kontext der Aufgabe beantwortet werden. Lediglich für spezielle Leistungsüberprüfungen wird das Feedback des Systems reduziert.

ALEA Mapping gibt den Lernenden weitere Hilfestellungen an die Hand. Mit dem Button „Show User Guide“ lassen sich Erläuterungen zur Benutzung des Systems anzeigen. Darüber hinaus können vordefinierte (in Abb. 4 obere Icons links) und empfohlene (in Abb. 4 untere Icons links) Hilfen in Form von Hilfetexten, Podcasts, Grafiken, Comics oder Videos erscheinen. Meldungen, die sich direkt auf die Bearbeitung beziehen, werden in Popup-Fenstern angezeigt. In einem Log (oben rechts) werden diese Meldungen aufgelistet. Hinweise zu neuen Empfehlungen und deren Anzahl werden dort ebenso angezeigt. Auf der linken Seite lässt sich Information über die Aufgaben aufklappen und anzeigen. Je nach der Art der ausgewählten Challenge können Aufgaben zur Wahl stehen oder es wird jeweils nächste angezeigt. Die Lernenden können (Button „Question \& Feedback“) jederzeit Fragen stellen oder Feedback eintragen.

\section{ALEA Normalisierung}

Übungsaufgaben zur Normalisierung auf Papier ergeben schwierig zu korrigierende Abgaben, da die Darstellungen der Studierenden zwar teilweise sehr gut sind, jedoch der überwiegende Teil schlecht lesbar und nicht sehr übersichtlich gestaltet ist. Hier ist eine Systemunterstützung für die Tutoren besonders hilfreich, neben den Vorteilen, die sich für die Lernenden ergeben. Die ALEA Normalisierung nutzt Anteile von ALEA Mapping, da hier ebenso Tabellen, Spalten, Constraints dargestellt und eingegeben werden müssen (siehe Abb. 6).

Zusätzlich ist es notwendig, die Vorgehensweise bei der Normalisierung abzubilden, so dass dies möglichst einfach durch die Tutoren und Lehrenden nachvollzogen und bewertet werden kann. Das bedeutet bspw., dass Tabellen, die zerlegt werden, deaktiviert werden müssen und Tabellen, die aus einer Tabellenzerlegung entstehen, die Information über die Ursprungstabelle enthalten sollten. Darüber hinaus ist möglichst viel Feedback an die Lernenden durch das System automatisiert zu berechnen. 


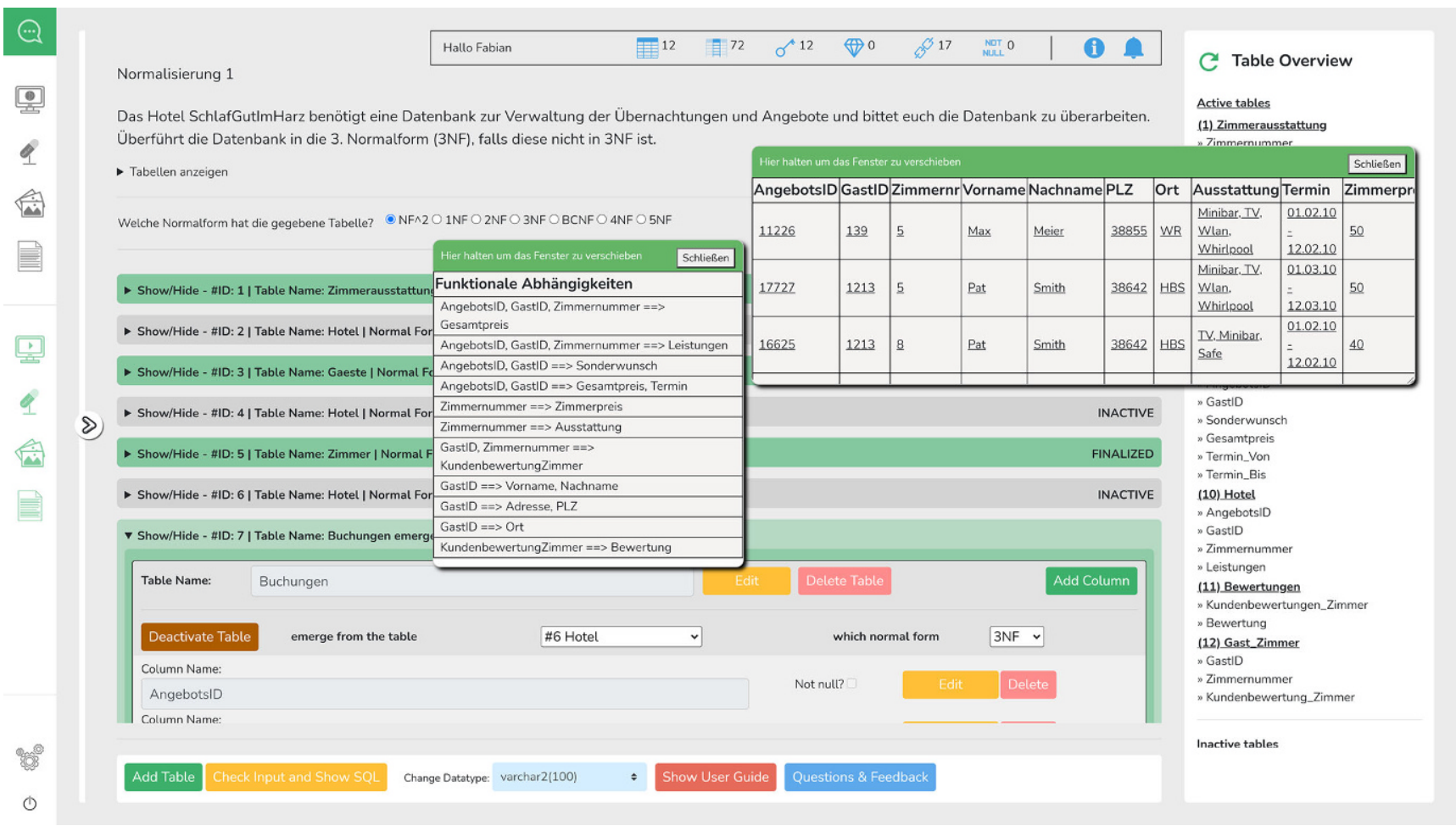

Abb. 6 Aufgabe zur Normalisierung (Ausschnitt)

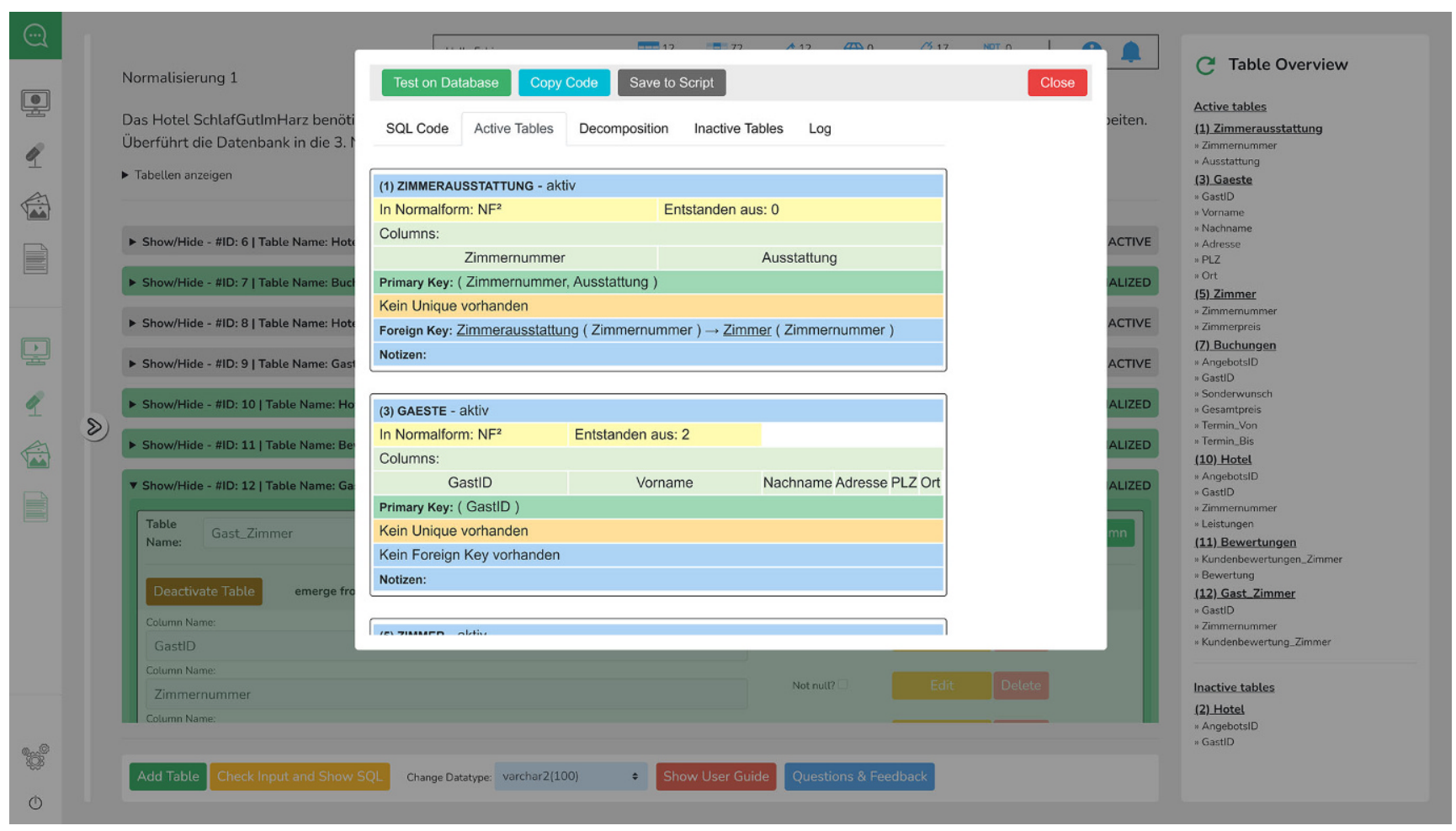

Abb. 7 Lösungsweg Normalisierung (Ausschnitt) 
Da entstehende Fremdschlüsselspalten mnemonisch benannt werden sollten, um Lesbarkeit, Verständlichkeit und Qualität des resultierenden Relationenmodells zu erhöhen und es dabei nicht nur eine Möglichkeit gibt, ist hier eine automatisierte Bewertung nicht vollständig möglich. Ob die Fremdschlüsselspalte entstanden ist, kann geprüft werden. Die Nachvollziehbarkeit ist mit der Speicherung geeigneter Information möglichst weitestgehend zu unterstützen. Es werden Darstellungen der Listen der aktiven Tabellen (siehe Abb. 7), der deaktivierten Tabellen und des vollständigen Ablaufs, der alle Tabellen auch die deaktivierten Tabellen enthält, bereitgestellt. Weitere Information kann sich zukünftig im Laufe der Verwendung als sinnvoll ergeben, so dass das System ergänzt und weiterentwickelt werden wird.

Die Lernenden können und sollen jeden Schritt begründen und erhalten Hilfestellungen, die je nach Kontext bspw. Testat oder Übungsphase unterschiedlich ausfallen. Bei der Eingabe der Lösung werden zudem die bereits im vorherigen Abschnitt beschriebenen Interaktionen mit dem System durchgeführt, so dass diese Regeln zum relationalen Modell eingehalten und geübt werden.

Aufgabenstellungen bestehen aus einem Aufgabentext, einer oder mehreren Tabellen in der Datenbank und einer Menge funktionaler Abhängigkeiten. Falls ein spezifischer Aufgabentext fehlt, kann ein generischer Aufgabentext verwendet werden. Es können sich zu einem Aufgabentext mit Tabellen durch unterschiedliche Mengen funktionaler Abhängigkeiten, die jedoch untereinander Äquivalenzmengen darstellen, mehrere Aufgaben ergeben, deren gültige Lösungen sich entsprechen. Die Nutzung dieser Variationen einer Aufgabenstellung kann den Lerneffekt erhöhen und gleichzeitig die Menge an verfügbaren Aufgaben ohne großen Aufwand erweitern. Zudem ist grundsätzlich ein Auswahlfeld zur Angabe der für die Tabellen in der Aufgabenstellungen geltenden Normalformen durch die Lernenden vorhanden. Da diese Information von den Aufgabenstellenden bereits in der Datenbank bereitgestellt wird, kann ein entsprechendes Feedback an die Lernenden generiert werden.

Die Information zu den Aufgaben wird in der Datenbank verwaltet, so dass hier eine komfortable administrative Komponente bei nächster Gelegenheit ergänzt werden wird. Insgesamt steht diese ALEA-Komponente im aktuellen Semester vor dem ersten praktischen Einsatztest. Hier werden sich Erkenntnisse zu Weiterentwicklungen ergeben. Die Datenbasis für die intelligenten Anteile des Systems muss entstehen, da die Daten aus den anderen Komponenten nicht alleine ausreichen. Zukünftig könnten aus größeren Tabellen in einer Datenbank über die automatische Berechnung von funktionalen Abhängigkeiten (bspw. DFD Algorithmus [1]) Aufgabenstellungen für diese ALEA-Komponente automatisch erzeugt werden. Ausschnitte aus denormalisierten Dimensionstabellen aus einem Star-Schema oder über Ver- bundoperationen berechnete Tabellen können als Basis für generierte Aufgaben dienen. Dies kann als Ergänzung für ein empfehlungsbasiertes Zuweisen von Aufgaben bspw. in Selbstlernphasen interessant sein.

\section{ALEA Frage-Antwort}

Für die Interaktion in den Lehrveranstaltungen werden Frage-Antwort-Komponenten des ALEA-Systems eingesetzt. Hier ist es möglich, synchron eine Frage an alle zu stellen oder asynchron einen Chat zu nutzen, um bspw. die Konzentration der Lernenden in den Laboren nicht zu stören.

Als Thema einer Gruppenarbeit im Rahmen einer früheren Lehrveranstaltung über anpassungsfähige Systeme war ein hybrides Empfehlungssystem auf der Basis einer Systemvorlage zu programmieren, welches das Stellen von Fragen durch Lehrende an alle angemeldeten Teilnehmenden ermöglicht. Deren Antworten sollten über einen Beamer bzw. einer Bildschirmfreigabe mit den Teilnehmenden diskutiert werden und direkt oder später durch die Fragestellenden bewertet werden können. Aus den gestellten Fragen mit deren bewerteten Antworten werden MultipleChoice-Fragen durch das System erstellt und den Lernenden als Übungsfragen entsprechend dem Antwortverhalten der Lernenden und dem gewünschten Schwierigkeitsgrad nacheinander zur Bearbeitung empfohlen. Die Ergebnisse dieser Projektaufgabe waren sehr erfolgreich. Eine weitere Komponente ist entstanden und konnte seitdem eingesetzt werden.

Darüber hinaus wird die allgemeine Frage-AntwortKomponente von ALEA sehr stark genutzt und stetig ergänzt und erweitert. Einzelne Aspekte werden zu eigenen jedoch im Gesamtsystem integrierten Komponenten, wie ALEA Normalisierung aus dem vorherigen Abschnitt.

Da sich Fragen der Lernenden nicht immer in den Präzensphasen ergeben und sich oft auf den Kontext der gerade durchgeführten Aufgabe beziehen, sind Möglichkeiten, um Fragen zu stellen in den Komponenten von ALEA an vielen Stellen bereits integriert und werden bei Bedarf ergänzt.

\section{Multimediale Elemente}

Vielfältige multimediale Elemente sind im Rahmen einer Masterarbeit [23] produziert worden. Der Schwerpunkt lag auf kurzen Erklärvideos, wie Screencasts. Zunächst wurde im Tonstudio der Hochschule aufgenommen, später aufgrund der Pandemie-Situation über Videokonferenzen. Die Screencasts sind unterschiedlich gestaltet. Einige enthalten bspw. Animationen oder Untertitel. Welche eine bessere Wirkung erzielen, müssen die Analysen ergeben. Mittlerweile produzierten die Tutoren eigenständig Screencasts 
mit jeweiliger Rücksprache zur beratenden Professorin, u.a. mehr als 50 für ELEA Mapping. Weitere Elemente wie Podcasts, Grafiken oder Hilfetexte werden ständig produziert und ergänzen das System. Die multimedialen Elemente werden entweder direkt Aufgaben oder Laboren zugeordnet oder über Empfehlungen für die Studierenden in ALEA zugänglich gemacht.

\section{Learning Analytics und intelligente Systeme}

Die gesamten Systeme sollen eine analytische Datenbasis speisen und es damit ermöglichen, intelligente Empfehlungen zu berechnen sowie Learning Analytics anzuwenden mit dem Ziel, den Lernprozess zu optimieren. Zudem sollen Analysen für Lehrpersonen bereitgestellt werden, so dass diese ihre Lehre optimieren können.

Im Rahmen einer Masterarbeit [3] wurden bereits Auswertungen durchgeführt. Die Abb. 8 zeigt als Beispiel die Anzahl der Versuche für eine SQL-Anfrage im ALEA-System. Erste Ergebnisse sind bereits vorhanden, jedoch ist es notwendig, dass die Datenbasis weiter anwächst. Für die Qualität der Empfehlungen ist dies ebenso notwendig.

\section{Diskussion und weitere Planung}

Interaktion mit den Lernenden über digitale Systeme, auch asynchron und trotzdem im Kontext der jeweiligen Bearbeitung, wird immer wichtiger, nicht nur in Selbstlernphasen, in berufsbegleitenden Studiengängen oder in Zeiten, die durch Umstände wie das Auftreten einer Pandemie die persönlichen Treffen einschränken.

Das Konzept der ACID-Transaktionen kann für Neulinge wirkungsvoll erfahren werden, wenn direkt gemeinsam auf einer Datenbank gearbeitet wird und dabei Wartezeiten durch Sperren auftreten (die auch im DBS dargestellt werden) oder die Sichtbarkeit für andere erst nach dem commit der Änderungen vorhanden ist. Anfragen werden vom DBMS abgelehnt, wenn Integritätsbedingungen nicht eingehalten werden oder Lernende warten müssen, bis dies entschieden werden kann. Effekte der Mehrbenutzerfähigkeit, Dauerhaftigkeit, Atomarität und Konsistenzsicherung lassen sich beispielhaft erleben. Diese gemeinsame Erfahrung ebnet das Grundverständnis, um die teilweise nicht leicht erfassbaren theoretischen Aspekte des ACID-Transaktionskonzepts, welche in der Vorlesung besprochen werden, gut und durchdringend nachvollziehen und verstehen zu können. Dies gilt umso mehr, da Datenbanken ein Fach darstellt, dessen Grundlagen in sehr vielen unterschiedlichen Studiengängen gelehrt werden, so dass Studierende mit sehr verschiedenen Vorkenntnissen zusammenkommen. Häufig müssen auch Masterstudierende die Einführungsvorlesung Datenbanken als Learning Agreement nachholen.

E-Learning-Systeme, welche Aspekte aus dem Bereich Datenbanken unterstützen, die bisher noch nicht umgesetzt wurden, werden angedacht. Zurzeit können diese Themen über Fragen mit Freitext-Antworten oder Multiple-ChoiceFragen geübt werden. Mit Analysen der Antworten können Einsichten gewonnen und geeignete Hilfestellungen ausgewählt oder berechnet empfohlen werden. Systeme, die das Üben von Lehrinhalten ermöglichen, können den Lernprozess optimieren. Dies gilt auch in der Präsenzlehre. Die positiven Effekte und Möglichkeiten sollen durch Learning

Abb. 8 Anzahl der Versuche für eine SQL-Aufgabe [3]

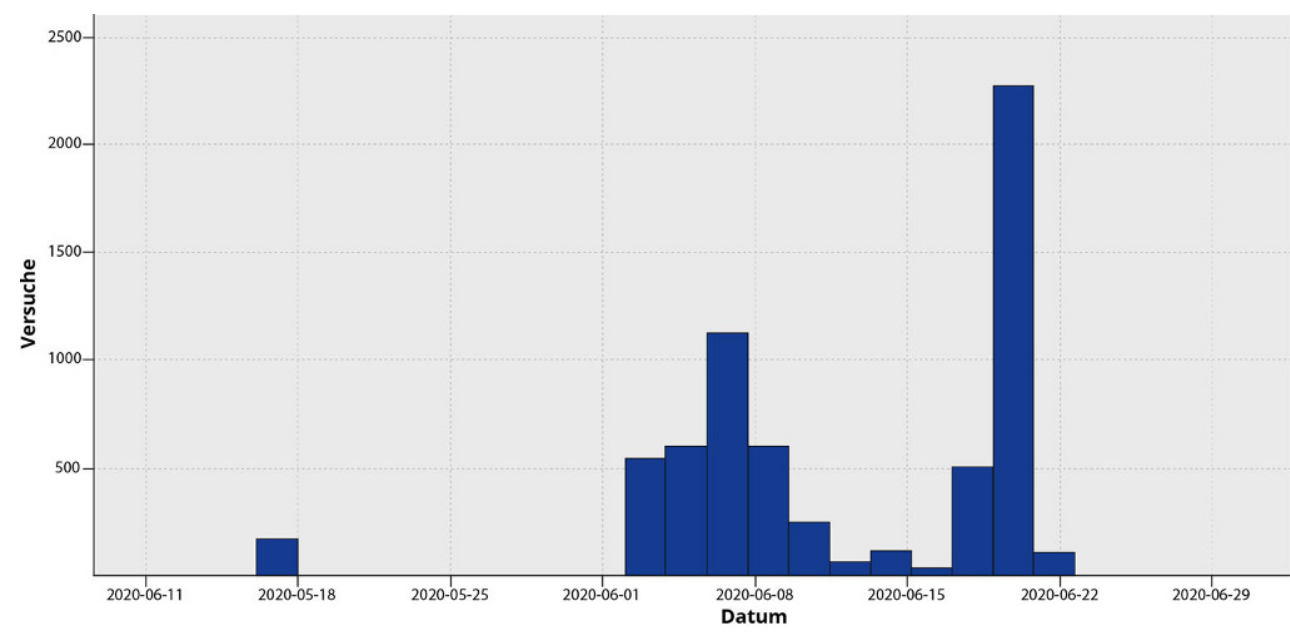

Höchste Anzahl an Versuchen: 78 (dann Aufgabe abgebrochen) Höchste Anzahl an Versuchen bis die Aufgabe geschafft wurde: 75 Anzahl der gestellen Abfragen: ca. 2600 
Analytics erfasst und durch entsprechende Weiterentwicklung der Systeme und Gestaltung der Lernprozesse genutzt werden.

\section{Zusammenfassung und Ausblick}

Nach einer Motivation für und Übersicht über eigenentwickelte E-Learning-Systeme und Anwendungen zum Erlernen von Themen aus dem Bereich Datenbanken an Hochschulen wurde das E-Learning-System ALEA beschrieben. Relevante Komponenten von ALEA zum Erlernen von SQL, ER-to-Relational-Mapping, Normalisierung sowie die allgemeine Frage-Antwort Komponente wurden dargestellt. Die Ergänzung des Systems durch die Produktion von vielfältigen digitalen Elementen, wie Screencasts oder Podcasts, welche im System als Hilfestellungen bereitgestellt werden, wurde erläutert. Diese Elemente können von den Lehrenden im Vorfeld zugeordnet oder durch das System empfohlen werden. Es wurde auf die Aspekte des Nutzens von Learning Analytics in ALEA zur Optimierung des Lernprozesses eingegangen.

ALEA wird von Lehrpersonen als auch im Rahmen von Teamprojekten, Hausarbeiten, Projekt-, Bachelor- und Masterarbeiten weiterentwickelt und ergänzt. Datenmodelle, Daten, Aufgaben, Hilfetexte und vieles mehr werden erstellt. Lehrvideos werden produziert. Die Studierenden stehen ALEA sehr positiv gegenüber und wünschen sich den weiteren Ausbau. Komponenten, die Ergebnisse von Analysen darstellen, werden erzeugt und die Datenbasis dafür erweitert. An der Intelligenz des Systems wird gearbeitet.

Beispiel-Videos und Zugänge zu ALEA sind über datencafe.hs-harz.de zu erreichen. Die meisten Komponenten stehen bisher lediglich im Intranet zur Verfügung.

Funding Open Access funding enabled and organized by Projekt DEAL.

Open Access Dieser Artikel wird unter der Creative Commons Namensnennung 4.0 International Lizenz veröffentlicht, welche die Nutzung, Vervielfältigung, Bearbeitung, Verbreitung und Wiedergabe in jeglichem Medium und Format erlaubt, sofern Sie den/die ursprünglichen Autor(en) und die Quelle ordnungsgemäß nennen, einen Link zur Creative Commons Lizenz beifügen und angeben, ob Änderungen vorgenommen wurden.

Die in diesem Artikel enthaltenen Bilder und sonstiges Drittmaterial unterliegen ebenfalls der genannten Creative Commons Lizenz, sofern sich aus der Abbildungslegende nichts anderes ergibt. Sofern das betreffende Material nicht unter der genannten Creative Commons Lizenz steht und die betreffende Handlung nicht nach gesetzlichen Vorschriften erlaubt ist, ist für die oben aufgeführten Weiterverwendungen des Materials die Einwilligung des jeweiligen Rechteinhabers einzuholen.

Weitere Details zur Lizenz entnehmen Sie bitte der Lizenzinformation auf http://creativecommons.org/licenses/by/4.0/deed.de.

\section{Literatur}

1. Abedjan Z, Schulz P, Naumann F (2014) DFD: Efficient Functional Dependency Discovery. CIKM '14: Proceedings of the 23rd ACM International Conference on Conference on Information and Knowledge Management November 2014 https://doi.org/101145, pp 949-958

2. Becher D (2012) DB-Normalizer. TU München. https://normalizer. db.in.tum.de/. Zugriffsdatum: 10.05.2021

3. Bittner C (2020) Optimierung des Lernprozesses durch Data Intelligence und Learning Analytics. Masterarbeit im Studiengang Technisches Innovationsmanagement, FBAI, Hochschule Harz

4. Chen P (1976) The-Entity-Relationship-Model. ACM transactions on database systems (TODS)

5. Dittrich J (2014) Die Umgedrehte Vorlesung - Chancen für die Informatiklehre. Datenbank-Spektrum 14, pp 69-76

6. Dittrich J (2016) Patterns in Data Management: A Flipped Textbook, 1st edn. CreateSpace Independent Publishing Platform

7. Faeskorn-Woyke H, Bertelsmeier B (2021) EDB - Das eLearning Datenbank Portal der TH Köln. https://edb2.gm.th-koeln.de/. Zugriffsdatum: 10.05 .202

8. Faeskorn-Woyke H, Bertelsmeier B, Gawenda D, N L (2008) edb -Ein studentisches Portal zur Unterstützung der Lehre im Fach Datenbanken. GI: Herbsttreffen der Fachgruppe DBS Heinrich-HeineUniversität Düsseldorf

9. Faeskorn-Woyke H, Bertelsmeier B, Gawenda D, N L (2008) edb - eLearning Datenbankportal der FH Köln. $\log$ OS2008 Osnabrück

10. Huth M (2008) Java Applikationsserver für den Produktiveinsatz. Projektarbeit im Studiengang Informatik, FBAI, Hochschule Harz

11. ILIAS open source e-Learning eV (2020) ILIAS The Open Source Learning Management System. https://www.ilias.de/. Zugriffsdatum: 10.05.2021

12. Kemper A, Neumann T (2021) SQL-Schnittstelle zu HyPer. TU München. http://hyper-db.com/interface.html. Zugriffsdatum: 10.05.2021

13. Kessler J, Tschuggnall M, Specht G (März 2019) In Proceedings of the 18th Fachtagung des GI-Fachbereichs Datenbanksysteme für Business, Technologie und Web (BTW) pp 503-506

14. Langer M (2016) Neuentwicklung eines SQL eLearning-Systems. Projektarbeit im Studiengang Informatik, FBAI, Hochschule Harz

15. Martin B (2008) Entwicklung einer Administrations-oberfläche für eine E-Learning Web-Applikation mit JavaServer Faces. Projektarbeit im Studiengang Informatik, FBAI, Hochschule Harz

16. Meyer H, Ritter N, Thor A, Nicklas D, Heuer A, Klettke M (2019) Datenbanksysteme für Business, Technologie und Web (BTW 2019) - Digitale Lehre im Fach Datenbanken - Workshopband

17. Mühe H (2012) IRA-Tool. TU München. https://ira.db.in.tum.de/. Zugriffsdatum: 10.05.2021

18. Rakow T, Faeskorn-Woyke H, Schiefer B, Vossen V, Wäsch J (2009) Tools für die Lehre im Fach Datenbanken. DatenbankSpektrum 9, pp 5-13

19. Schildgen J (2018) Sprachkurs SQL. Das Datenbanken-Hörbuch

20. Schildgen J (2020) MonstER Park - The Entity-Relationship-Diagram Learning Game. ER Forum. http://ceur-ws.org/Vol-2716/ paper13.pdf

21. Schildgen J (2021) MonstER Park. OTH Regensburg. https://www. monst-er.de/. Zugriffsdatum: 10.05.2021

22. Schildgen J, Deßloch S (2013) „Gib mir so viel Gold, wie die Metzger im Nachbardorf zusammen besitzen und ich lasse den Piloten frei!“ - Spielbasiertes Lernen von SQL-Grundlagen. DatenbankSpektrum 1(3), pp 243-249

23. Schorch F (2020) Multimediale Elemente für ein intelligentes E-Learning-System. Master's thesis, Masterarbeit im Studiengang Medien- und Spielekonzeption, FBAI, Hochschule Harz 
24. Specht G, Kessler J, Mayerl M, Tschuggnall M (2021) RelaX. Datenbank Spektrum 21, pp 49—54, https://doi.org/10.1007/s13222021-00367-x

25. Thor A (2019) Ein Blended Learning Kurs für das Fach Datenbanksysteme. HWTK Leipzig. https://dbs.uni-leipzig.de/file/20190304_
BTW_Workshop_Digitale_Lehre_Datenbanken.pdf. Zugriffsdatum: 10.05.2021 